PROCEEDINGS OF THE

AMERICAN MATHEMATICAL SOCIETY

Volume 131, Number 11, Pages 3547-3551

S 0002-9939(03)06900-4

Article electronically published on February 14, 2003

\title{
CIRCLE MAPS HAVING AN INFINITE $\omega$-LIMIT SET WHICH CONTAINS A PERIODIC ORBIT HAVE POSITIVE TOPOLOGICAL ENTROPY
}

\author{
NAOTSUGU CHINEN \\ (Communicated by Ronald A. Fintushel)
}

\begin{abstract}
Let $f$ be a continuous map from the circle to itself. The main result of this paper is that the topological entropy of $f$ is positive if and only if $f$ has an infinite $\omega$-limit set which contains a periodic orbit.
\end{abstract}

\section{INTRODUCTION}

Let $f$ be a continuous map from a continuum $X$ to itself. We denote the $n$-fold composition $f^{n}$ of $f$ with itself by $f \circ \ldots \circ f$ and $f^{0}$ the identity map. Let $x$ be a point of $X$. We define the orbit $\operatorname{Orb}(x, f)$ of $x$ by $\left\{f^{n}(x) \mid n \geq 0\right\}$, and we define the $\omega$-limit set of $x$ to be the set $\omega(x, f)=\{y \in X \mid$ for each neighborhood $V$ of $y$ and each positive integer $n, V \cap \operatorname{Orb}\left(f^{n}(x), f\right)$ is nonempty $\}$. It is known that $\omega(x, f)$ is nonempty and strongly invariant, i.e. $f(\omega(x, f))=\omega(x, f)$. See [BC] p.72] for details. If $\omega(x, f)$ is finite, by [BC] Lemma IV4, p.72], $\omega(x, f)$ is a periodic orbit of some point.

Let $z$ be a periodic point of $f$. The unstable set of $z$ is defined to be the set $W(z, f)=\left\{x \in X \mid\right.$ for any neighborhood $V$ of $z, x \in f^{k}(V)$ for some $\left.k>0\right\}$. A point $y$ is homoclinic for $f$ if there exists a point $z \neq y$ such that $f^{n}(z)=z$ for some $n>0, y \in W\left(z, f^{n}\right)$ and $f^{k n}(y)=z$ for some $k>0$. This definition of homoclinic points first appeared in $[\mathrm{B}$. A point $x \in X$ is a nonwandering point for $f$ if for any open set $U$ containing $x$ there exists $n>0$ such that $f^{n}(U) \cap U \neq \emptyset$.

The following theorem is well known.

Theorem 1.1. Let $f$ be a continuous map from a compact interval I to itself. The following statements are equivalent:

(a) $f$ has positive topological entropy,

(b) $f^{n}$ is strictly turbulent for some positive integer $n$,

(c) $f$ has a nonwandering homoclinic point, and

(d) for some $c \in I, \omega(c, f)$ properly contains a periodic orbit.

See [BC, p.25] for strictly turbulent, [BC, Section VIII] for topological entropy and [BC, p.124, p.153 and p.218] or [SKSF, Theorem 4.19, p.112] for Theorem 1.1.

Received by the editors April 15, 2002 and, in revised form, June 24, 2002.

2000 Mathematics Subject Classification. Primary 37B40, 37E10; Secondary 28D05, 54H20.

Key words and phrases. $\omega$-limit set, circle, unstable set, homoclinic point, nonwandering point, topological entropy. 
We can consider the following theorem, analogous to the corresponding conditions in Theorem 1.1.

Theorem 1.2. Let $f$ be a continuous map from the circle $S^{1}$ to itself. The following statements are equivalent:

(a) $f$ has positive topological entropy,

(b) $f^{n}$ is strictly turbulent for some positive integer $n$,

(c) $f$ has a nonwandering homoclinic point, and

(d) for some $c \in S^{1}, \omega(c, f)$ properly contains a periodic orbit.

See [BC, p.229] for strictly turbulent of circle maps. Although the aim of this paper is to prove Theorem 1.2, it is known that $(a),(b)$ and $(c)$ are equivalent (see [BC, p.229] or [BCMN, Theorem B+, p.529] for details) and that conditions $(a),(b)$ and $(c)$ imply condition $(d)$ (see [BC, p.230] for details). Therefore, in this paper, we prove that condition $(d)$ implies condition $(a)$. This is the answer of the question in [BC, p.230].

\section{Definitions}

Notation 2.1. Let $Y$ be a subspace of a space $X$, and let int $Y$ and $\mathrm{Cl} Y$ denote the interior and the closure of $Y$ in a space $X$, respectively.

Definition 2.2. Let $f$ be a continuous map from a space $X$ to itself. A point $x \in X$ is a fixed point for $f$ if $f(x)=x$. A point $x \in X$ is a periodic point of period $n \geq 1$ for $f$ if $f^{n}(x)=x$. We denote the sets of fixed points, periodic points and nonwandering points for $f$ by $\mathrm{F}(f), \mathrm{P}(f)$ and $\Omega(f)$, respectively.

Definition 2.3. Let us denote a subspace $\{z|| z \mid=1\}$ of the complex plane, i.e., the circle, by $S^{1}$. Let $x$ and $y$ be two distinct points of $S^{1}$. We denote the closed arc from $x$ counterclockwise to $y$ by $[x, y]$, and we denote $(x, y)=[x, y] \backslash\{x, y\}$, $(x, y]=[x, y] \backslash\{x\}$ and $[x, y)=[x, y] \backslash\{y\}$.

Let $\pi$ be the canonical projection from the real line onto $S^{1}$ defined by $\pi(t)=$ $e^{2 \pi i t}, \widetilde{x}$ and $\widetilde{y}$ two points of the real line such that $\widetilde{y} \in(\widetilde{x}, \widetilde{x}+1), \pi(\widetilde{x})=x$ and $\pi(\widetilde{y})=$ $y$. We see that $\pi \mid[\widetilde{x}, \widetilde{y}]:[\widetilde{x}, \widetilde{y}] \rightarrow[x, y]$ is a homeomorphism. Every continuous map $f$ from the circle $S^{1}$ to itself has countable many lifts, i.e., continuous maps $\tilde{f}$ from the real line to itself satisfying $f \circ \pi=\pi \circ \widetilde{f}$.

Definition 2.4. Let $p$ be a fixed point of a continuous map $f$ from $S^{1}$ to itself. If $V$ is a neighborhood of $p$ in $[p,-p)$ (in $(-p, p]$, respectively), we say $V$ is an $R$ neighborhood of $p$ (L-neighborhood of $p$, respectively). Let $S=R, L$. The $S$-sided unstable manifold of $p$ is defined by

$W(p, f, S)=\left\{x \in S^{1} \mid\right.$ for any $S$-neighborhood $V$ of $p, x \in f^{k}(V)$ for some $\left.k>0\right\}$.

Let $\widetilde{p}$ be a fixed point of a continuous map $\widetilde{f}$ from the real line to itself. If $\widetilde{V}$ is a neighborhood of $\widetilde{p}$ in $[\widetilde{p}, \widetilde{p}+1)$ (in $(\widetilde{p}-1, \widetilde{p}]$, respectively), we say $\widetilde{V}$ is an $R$-neighborhood of $\widetilde{p}$ (L-neighborhood of $\widetilde{p}$, respectively). The S-sided unstable manifold of $\widetilde{p}$ is defined by $W(\widetilde{p}, \widetilde{f}, S)=\{\widetilde{x} \mid$ for any $S$-neighborhood $\widetilde{V}$ of $\widetilde{p}, \widetilde{x} \in$ $\widetilde{f}^{k}(\widetilde{V})$ for some $\left.k>0\right\}$.

We see that $W(p, f)=W(p, f, R) \cup W(p, f, L)$ and that $W(\widetilde{p}, \widetilde{f})=W(\widetilde{p}, \widetilde{f}, R) \cup$ $W(\widetilde{p}, \widetilde{f}, L)$. 


\section{Elementary Lemmas}

By [BC, Proposition II 2, p.48], we have the following lemma.

Lemma 3.1. Let $\widetilde{f}$ be a continuous map from the real line to itself and $\widetilde{z} \in \mathrm{F}(\widetilde{f})$.

(1) If $W(\widetilde{z}, \widetilde{f}, L) \cap(\widetilde{z}, \infty) \neq \emptyset$, then $W(\widetilde{z}, \widetilde{f}, R) \subset W(\widetilde{z}, \widetilde{f}, L)$.

(2) If $W(\widetilde{z}, \widetilde{f}, L) \cap(-\infty, \widetilde{z})=\emptyset$, then $W(\widetilde{z}, \widetilde{f}, L)=\{\widetilde{z}\}$ or $W(\widetilde{z}, \widetilde{f}, R)$.

(3) If $W(\widetilde{z}, \widetilde{f}, R) \cap(-\infty, \widetilde{z}) \neq \emptyset$, then $W(\widetilde{z}, \widetilde{f}, L) \subset W(\widetilde{z}, \widetilde{f}, R)$.

(4) If $W(\widetilde{z}, \widetilde{f}, R) \cap(\widetilde{z}, \infty)=\emptyset$, then $W(\widetilde{z}, \widetilde{f}, L)=\{\widetilde{z}\}$ or $W(\widetilde{z}, \widetilde{f}, R)$.

By [BC, Proposition II 1 and 3] and [BCMN, Lemma 1], we have the following lemma.

Lemma 3.2. Let $X$ be either a compact interval or the circle or the real line, $f$ a continuous map from $X$ to itself, $z \in \mathrm{F}(f)$ and $S=R, L$. Then $W(z, f, S)$ and $W(z, f)$ are connected, $f(W(z, f, S))=W(z, f, S)$ and $f(W(z, f))=W(z, f)$.

Lemma 3.3. Let $f$ be a continuous map from the circle $S^{1}$ to itself, $z \in \mathrm{F}(f)$ and $S=R, L$. Also, let $\widetilde{f}$ be the lift of $f$ with $\widetilde{z} \in \mathrm{F}(\widetilde{f})$ satisfying $\pi(\widetilde{z})=z$. Then $\pi(W(\widetilde{z}, \widetilde{f}, S))=W(z, f, S)$ and $\pi(W(\widetilde{z}, \widetilde{f}))=W(z, f)$.

Proof. We give the proof for the first assertion. Let $\widetilde{x} \in W(\widetilde{z}, \widetilde{f}, S)$ and $U$ a small $S$-neighborhood of $z$. There exists a small $S$-neighborhood $\widetilde{V}$ of $\widetilde{z}$ with $\pi(\widetilde{V}) \subset U$. Since $\widetilde{x} \in W(\widetilde{z}, \widetilde{f}, S)$, we have a positive integer $n$ such that $\widetilde{x} \in \widetilde{f}^{n}(\widetilde{V})$, thus, $\pi(\widetilde{x}) \in \pi\left(\tilde{f}^{n}(\widetilde{V})\right)=f^{n}(\pi(\widetilde{V})) \subset f^{n}(U)$. We conclude that $\pi(\widetilde{x}) \in W(z, f, S)$ and that $\pi(W(\widetilde{z}, \widetilde{f}, S)) \subset W(z, f, S)$.

Let $x \in W(z, f, S) \backslash\{z\}$ and let $\left\{\widetilde{U_{n}}\right\}_{n=1}^{\infty}$ be a sequence of small connected $S$ neighborhoods of $\widetilde{z}$ with $\bigcap_{n=1}^{\infty} \widetilde{U_{n}}=\{\widetilde{z}\}$. Since $\pi\left(\widetilde{U_{n}}\right)$ is a small $S$-neighborhood of $z$ for each $n$, there exists a positive integer $k_{n}$ such that $x \in f^{k_{n}}\left(\pi\left(\widetilde{U_{n}}\right)\right)$. Since $x \in \pi\left(\widetilde{f}^{k_{n}}\left(\widetilde{U_{n}}\right)\right)$, we see that $\pi^{-1}(x) \cap \widetilde{f}^{k_{n}}\left(\widetilde{U_{n}}\right) \neq \emptyset$. Thus there exist $\widetilde{x} \in \pi^{-1}(x) \cap$ $(\widetilde{z}-1, \widetilde{z}+1)$ and a sequence $\ell_{1}, \ell_{2}, \ldots$ of positive integers such that $\widetilde{x} \in \widetilde{f}^{k_{\ell_{n}}}\left(\widetilde{U_{\ell_{n}}}\right)$ for each $n$. This shows that $\widetilde{x} \in W(\widetilde{z}, \widetilde{f}, S)$ and that $\pi(W(\widetilde{z}, \widetilde{f}, S)) \supset W(z, f, S)$.

Corollary 3.4. Let $f$ be a continuous map from the circle $S^{1}$ to itself with lift $\tilde{f}$. If $\widetilde{y}$ is a homoclinic point for $\widetilde{f}$, then $y=\pi(\widetilde{y})$ is a homoclinic point for $f$.

Lemma 3.5. Let $f$ be a continuous map from the circle $S^{1}$ to itself with lift $\tilde{f}$ and $\widetilde{z} \in \mathrm{F}(\widetilde{f}), S, S^{\prime} \in\{R, L\}$, and $\widetilde{y}, \widetilde{z}^{\prime}$ two points of the real line satisfying that $\widetilde{y} \in W(\widetilde{z}, \widetilde{f}, S), \pi(\widetilde{z})=\pi\left(\widetilde{z}^{\prime}\right) \neq \pi(\widetilde{y})$ and $\widetilde{f}^{n}(\widetilde{y})=\widetilde{z}^{\prime}$ for some $n \geq 1$. If $\widetilde{f}^{n}(\widetilde{U})$ contains an $S$-neighborhood of $\widetilde{z}^{\prime}$ for each $S^{\prime}$-neighborhood $\widetilde{U}$ of $\widetilde{y}$, then $y=\pi(\widetilde{y})$ is a nonwandering homoclinic point for $f$.

Proof. We notice that $z=\pi(\widetilde{z}) \in \mathrm{F}(f)$ and that $f^{n}(y)=\pi\left(\tilde{f}^{n}(\widetilde{y})\right)=\pi\left(\widetilde{z}^{\prime}\right)=z$. It follows from Lemma 3.3 that $y \in \pi(W(\widetilde{z}, \widetilde{f}, S))=W(z, f, S) \subset W(z, f)$. It suffices to show that $y \in \Omega(f)$. Let $U$ be a small $S^{\prime}$-neighborhood of $y$ and $\widetilde{U}$ an $S^{\prime}$-neighborhood of $\widetilde{y}$ with $\pi(\widetilde{U})=U$. From the assumption, $\widetilde{f}^{n}(\widetilde{U})$ contains some small $S$-neighborhood $\widetilde{V}$ of $\widetilde{z}^{\prime}$. Since $\pi(\widetilde{z})=\pi\left(\widetilde{z}^{\prime}\right)$, there exists the integer $\delta$ such that $\widetilde{z}^{\prime}=\widetilde{z}+\delta$. Set $\widetilde{V}-\delta=\{\widetilde{x}-\delta \mid \widetilde{x} \in \widetilde{V}\}$. We notice that $\widetilde{V}-\delta$ is an $S$-neighborhood of $\widetilde{z}$ and that $\pi(\widetilde{V}-\delta)=\pi(\widetilde{V})$ is an $S$-neighborhood of $z$. Also, since $\widetilde{y} \in W(\widetilde{z}, \widetilde{f}, S)$, we have a positive integer $m$ such that $\widetilde{y} \in \widetilde{f}^{m}(\widetilde{V}-\delta)$. This 
shows that $y \in \pi\left(\tilde{f}^{m}(\widetilde{V}-\delta)\right)=f^{m}(\pi(\widetilde{V}-\delta))=f^{m}(\pi(\widetilde{V})) \subset f^{m}\left(\pi\left(\tilde{f^{n}}(\widetilde{U})\right)\right)=$ $f^{m+n}(\pi(\widetilde{U}))=f^{m+n}(U)$, thus, $y \in \Omega(f)$.

\section{A proof of Theorem 1.2}

Lemma 4.1. Let $f$ be a continuous map from the circle $S^{1}$ to itself. If there exists a point $c \in S^{1}$ such that $\omega(c, f)$ is infinite containing some fixed point, then $f$ has positive topological entropy.

Proof. Set $c_{m}=f^{m}(c)$ for each $m$. Choose $z \in \omega(c, f) \cap \mathrm{F}(f)$. We have an increasing sequence $\left\{n_{k}\right\}$ of positive integers, $S=R, L$ and an $S$-neighborhood $U_{z}$ of $z$ such that $c_{n_{k}} \in U_{z} \backslash\{z\}$ for all $k$ and that $\lim _{k \rightarrow \infty} c_{n_{k}}=z$. Since $\omega(c, f)$ is infinite, we see that $W(z, f, S) \neq\{z\}$.

We show that $\operatorname{Orb}(c, f) \cap W(z, f, S) \neq \emptyset$. We suppose that $\operatorname{Orb}(c, f) \cap W(z, f, S)$ $=\emptyset$. Since $W(z, f, S)$ is connected by Lemma 3.2, there exist a point $x$ of $\omega(c, f)$ and a compact interval $A$ such that $\omega(c, f) \subset A, A \backslash \operatorname{int} A=\{z, x\}$ and $W(z, f, S) \subset$ $S^{1} \backslash \operatorname{int} A$. Since $\omega(c, f)$ is infinite, by the definition of $W(z, f, S)$, there exists a point $x^{\prime} \in \mathrm{Cl} W(z, f, S) \cap \operatorname{int} A$. This is a contradiction.

Since $\operatorname{Orb}(c, f) \cap W(z, f, S) \neq \emptyset$, Lemma 3.2 implies that $\omega(c, f) \subset \mathrm{Cl} W(z, f, S)$. We suppose that $\mathrm{Cl} W(z, f, S) \neq S^{1}$. By Lemma 3.2, we see that $\mathrm{Cl} W(z, f, S)$ is a compact interval. Since $\operatorname{Orb}(c, f) \cap W(z, f, S) \neq \emptyset$, we have $c_{m} \in W(z, f, S)$ for some $m$. Since $\omega\left(c_{m}, f\right)=\omega(c, f)$ and $f(\mathrm{Cl} W(z, f, S))=\mathrm{Cl} W(z, f, S)$, we have $\omega(c, f)=\omega\left(c_{m}, f \mid \mathrm{Cl} W(z, f, S)\right)$, where $f \mid \mathrm{Cl} W(z, f, S): \mathrm{Cl} W(z, f, S) \rightarrow$ $\mathrm{Cl} W(z, f, S)$ is the restriction of $f$. From Theorem 1.1, $f \mid \mathrm{Cl} W(z, f, S)$ has positive topological entropy. We conclude from [BC] Proposition VIII 5, p.193] that $f$ has positive topological entropy. We may assume that $\mathrm{Cl} W(z, f, S)=S^{1}$.

Since $(a)$ and $(c)$ in Theorem 1.2 are equivalent, we are going to show that $f$ has a nonwandering homoclinic point.

Let $\tilde{f}$ be the lift of $f$ with $\widetilde{z} \in \mathrm{F}(\widetilde{f})$ satisfying $\pi(\widetilde{z})=z$. We suppose that $\mathrm{Cl} W(\widetilde{z}, \widetilde{f}, S)$ is compact, i.e., bounded. Since $\pi(\mathrm{Cl} W(\widetilde{z}, \widetilde{f}, S))=\mathrm{Cl} W(z, f, S)=S^{1}$ by Lemma 3.3 , there exists a point $\widetilde{c} \in \mathrm{Cl} W(\widetilde{z}, \widetilde{f}, S)$ satisfying $\pi(\widetilde{c})=c$. Set $\widetilde{c_{m}}=\widetilde{f}{ }^{m}(\widetilde{c})$ for each $m$. Let $\widetilde{f} \mid \mathrm{Cl} W(\widetilde{z}, \widetilde{f}, S): \mathrm{Cl} W(\widetilde{z}, \widetilde{f}, S) \rightarrow \mathrm{Cl} W(\widetilde{z}, \widetilde{f}, S)$ be the restriction of $\widetilde{f}$. Since $\pi\left(\widetilde{c_{m}}\right)=c_{m}$ for each $m$, we have $\pi(\omega(\widetilde{c}, \widetilde{f} \mid \mathrm{Cl} W(\widetilde{z}, \widetilde{f}, S))) \subset$ $\omega(c, f)$. Let $x \in \omega(c, f)$. We have a sequence $n_{1}, n_{2}, \ldots$ of positive integers such that $\lim _{k \rightarrow \infty} c_{n_{k}}=x$. Since $\mathrm{Cl} W(\widetilde{z}, \tilde{f}, S)$ is compact, there exist a subsequence $n_{i_{1}}, n_{i_{2}}, \ldots$ and $\widetilde{x} \in \operatorname{Cl} W(\widetilde{z}, \widetilde{f}, S)$ such that $\lim _{k \rightarrow \infty} \widetilde{c_{n_{i_{k}}}}=\widetilde{x}$. Since $\widetilde{x} \in \omega(\widetilde{c}, \widetilde{f} \mid \mathrm{Cl} W(\widetilde{z}, \tilde{f}, S))$ and $\pi(\widetilde{x})=x$, we see that $\pi(\omega(\widetilde{c}, \widetilde{f} \mid \mathrm{Cl} W(\widetilde{z}, \tilde{f}, S))) \supset$ $\omega(c, f)$ and conclude that $\pi(\omega(\widetilde{c}, \widetilde{f} \mid \mathrm{Cl} W(\widetilde{z}, \widetilde{f}, S)))=\omega(c, f)$. This shows that $\omega(\widetilde{c}, \widetilde{f} \mid \mathrm{Cl} W(\widetilde{z}, \widetilde{f}, S))$ properly contains a periodic orbit. By Theorem 1.1, $\widetilde{f} \mid \mathrm{Cl} W(\widetilde{z}, \tilde{f}, S)$ has a homoclinic point $\widetilde{y} \in \Omega(\widetilde{f} \mid \mathrm{Cl} W(\widetilde{z}, \tilde{f}, S))$. Since $\pi(\Omega(\widetilde{f} \mid \mathrm{Cl} W(\widetilde{z}, \tilde{f}, S))) \subset \pi(\Omega(\widetilde{f})) \subset \Omega(f)$, by Corollary 3.4, $f$ has a homoclinic point $\pi(\widetilde{y}) \in \Omega(f)$. We may assume that $\mathrm{ClW}(\widetilde{z}, \widetilde{f}, S)$ is unbounded.

We suppose that $W(\widetilde{z}, \widetilde{f}, S)$ contains some small open connected $S$-neighborhood $\widetilde{U}$ of $\widetilde{z}$. Since $\operatorname{Cl} W(\widetilde{z}, \widetilde{f}, S)$ is unbounded containing $\widetilde{z}$, we have $\delta=1,-1$ such that $\widetilde{z}+\delta \in W(\widetilde{z}, \widetilde{f}, S)$. We suppose that $S=L$. Since $\widetilde{z}+\delta \in W(\widetilde{z}, \widetilde{f}, L)$, there exists a positive integer $n$ such that $\widetilde{z}+\delta \in \widetilde{f}^{n}(\widetilde{U})$. Set $\widetilde{y}=\max \left\{\widetilde{y}^{\prime} \in \widetilde{U} \mid \widetilde{f}^{n}\left(\widetilde{y}^{\prime}\right)=\widetilde{z}+\delta\right\}$. 
If $\delta=1$, we see that $\tilde{f}^{n}(\widetilde{V})$ is an $L$-neighborhood of $\widetilde{z}+1$ for each small $R$ neighborhood $\widetilde{V}$ of $\widetilde{y}$. It follows from Lemma 3.5 that $y=\pi(\widetilde{y})$ is a nonwandering homoclinic point.

Next we suppose that $\delta=-1$ and $\widetilde{z}+1 \notin W(\widetilde{z}, \widetilde{f}, L)$. Since $(-\infty, \widetilde{z}-1) \subset$ $W(\widetilde{z}, \widetilde{f}, L)$, there exist a point $\widetilde{x} \in(\widetilde{z}-1, \widetilde{z})$ and a positive integer $m$ such that $\widetilde{f}^{m}(\widetilde{x})<\widetilde{z}-1$. Thus, we have $\widetilde{y}^{\prime}=\min (\widetilde{x}, \widetilde{z}) \cap \widetilde{f}^{-m}(\widetilde{z}-1)$. By the definition of $\widetilde{y}^{\prime}$, we see that $\tilde{f}^{m}\left(\widetilde{V}^{\prime}\right)$ is an $L$-neighborhood of $\widetilde{z}-1$ for each small $L$-neighborhood $\widetilde{V}^{\prime}$ of $\widetilde{y}^{\prime}$. It follows from Lemma 3.5 that $y^{\prime}=\pi\left(\widetilde{y}^{\prime}\right)$ is a nonwandering homoclinic point.

We can prove $S=R$ by an argument similar to that for $S=L$. Thus, we may assume that $W(\widetilde{z}, \widetilde{f}, S)$ contains no $S$-neighborhood of $\widetilde{z}$.

Without loss of generality, we may assume that $S=L$. We see from Lemma $3.1(2)$ that $W(\widetilde{z}, \widetilde{f}, R)=W(\widetilde{z}, \widetilde{f}, L)=[\widetilde{z}, \infty)$. Let $\widetilde{U}$ be a small connected $R$ neighborhood of $\widetilde{z}$. Since $\widetilde{z}+1 \in W(\widetilde{z}, \widetilde{f}, R)$, there exists a positive integer $n$ such that $\widetilde{z}+1 \in \widetilde{f}^{n}(\widetilde{U})$. Set $\widetilde{y}=\min \left\{\widetilde{y}^{\prime} \in \widetilde{U} \mid \widetilde{f}^{n}\left(\widetilde{y}^{\prime}\right)=\widetilde{z}+1\right\}$. As above, we can show that $y=\pi(\widetilde{y})$ is a nonwandering homoclinic point.

Theorem 4.2. Condition $(d)$ in Theorem 1.2 implies condition $(a)$ in Theorem 1.2 .

Proof. Let $f$ be a continuous map from the circle $S^{1}$ to itself. Let $c \in S^{1}$ such that $\omega(c, f)$ properly contains a periodic point of period $n$. By BC, Lemma IV 4 , p.72], $\omega(c, f)$ is infinite, thus, $\omega\left(f^{j}(c), f^{n}\right)$ is also infinite for all $j$ with $0 \leq j<n$ by $\left[\mathrm{BC}\right.$ p.70]. Since at least one of these $\omega$-limit sets contains a fixed point of $f^{n}$ by [BC, p.70], we see from Lemma 4.1 that $f^{n}$ has positive topological entropy. We conclude from $[\mathrm{BC}$. Proposition VIII 2, p.191] that $f$ has positive topological entropy.

\section{REFERENCES}

[B] L. Block, Homoclinic points of mappings of the interval, Proc. Amer. Math. Soc. 72 (1978), 576-580. MR 81m:58063

[BC] L. Block and W. Coppel, Dynamics in One Dimension, Lecture Notes in Math. 1513, Springer-Verlag, 1992. MR 93g:58091]

[BCMN] L. Block, E. Coven, I. Mulvey and Z. Nitecki, Homoclinic and non-wandering points for maps of the circle, Ergodic Theory Dynam. Systems, 3 (1983), 521-532. MR 86b:58101

[SKSF] A. Sharkovsky, S. Kolyada, A. Sivak, and V. Fedorenko, Dynamics of one-dimensional maps, Translated from the 1989 Russian original, Math. and its Appl., 407. Kluwer Academic Publishers Group, Dordrecht, 1997. MR 98k:58083

Institute of Mathematics, University of TsukUba, IBRAKi 305-8571, JAPAN

E-mail address: naochin@math.tsukuba.ac.jp 